\title{
Envelhecimento com dependência: responsabilidades e demandas da família
}

\author{
Aging with dependence: \\ family needs and responsibilities
}

Célia Pereira Caldas 1,2

\footnotetext{
1 Universidade Aberta da Terceira Idade, Universidade do Estado do Rio de Janeiro. Rua São Francisco Xavier 524, Rio de Janeiro, $R J$ 20559-900, Brasil.

2 Faculdade de Enfermagem, Universidade do Estado do Rio de Janeiro. Boulevard 28 de Setembro 157, Rio de Janeiro, $R J$ 20551-030, Brasil.
}

\begin{abstract}
This article highlights the phenomenon of "aging with dependence" as a challenge to be dealt with by the field of collective health and discusses responsibilities for providing health care to this population contingent, comparing the necessary and existing conditions for families to assume responsibility for such care. The analysis is based on authors and theoretical references involving studies on aging, dependence, public policies, and available community resources for health care for the elderly. The following themes are emphasized: dependence and aging, the economic issue of dependence, family care, and family needs. The article concludes that since dependence is a dynamic process, it should be approached through programs that range from health promotion strategies to the establishment of support networks for long-term care in the community. These programs should be part of a public policy that involves all sectors of society and that can provide back-up for dependent seniors, with or without family support.
\end{abstract}

Key words Health; Aging; Home Nursing

Resumo Os objetivos deste artigo são apontar o fenômeno "envelhecimento com dependência" como um desafio a ser incorporado pelo campo da Saúde Coletiva e discutir a responsabilidade pela assistência a esse contingente populacional, contrapondo as condições necessárias às existentes para que as famílias assumam os cuidados. A análise é feita a partir de autores e marcos teóricos que desenvolveram estudos sobre envelhecimento, dependência, políticas públicas e recursos comunitários disponíveis para a atenção à saúde do idoso. Os temas destacados para esta análise foram: a dependência e o envelhecimento; a questão econômica da dependência; o cuidado familiar; e as necessidades da família. Conclui-se que, por ser um processo dinâmico, a dependência deve ser abordada por intermédio de programas que incluam desde estratégias de promoção da saúde até o estabelecimento de redes de apoio a cuidados de longa duração na comunidade. Tais programas devem fazer parte de uma política pública que envolva todos os setores da sociedade e que possa respaldar os idosos dependentes, com ou sem suporte familiar. Palavras-chave Saúde do Idoso; Envelhecimento; Cuidados Domiciliares de Saúde 


\section{Introdução}

Alicerçado em conteúdo teórico-conceitual na área do envelhecimento e visando a contribuir para o campo da Saúde Coletiva, o presente estudo tem como eixo a questão do envelhecimento com dependência e o impacto que esta responsabilidade gera na família. A intenção é a de, por meio de uma revisão crítica sobre o tema, dar subsídios para discussões que levem a medidas de apoio aos idosos e suas famílias.

A escolha do tema deu-se em função da necessidade de reconhecer que essa questão tende a se tornar um problema de saúde pública. É crescente o contingente de idosos dependentes, uma vez que a expectativa de vida vem aumentando.

Os objetivos que este trabalho procura atingir são, portanto, apontar o fenômeno "envelhecimento com dependência" como um desafio a ser incorporado pelo campo da Saúde Pública e contrapor duas situações: (a) as condições necessárias à manutenção dos cuidados com as pessoas idosas e dependentes na comunidade; e (b) as condições que a família realmente dispõe no meio urbano brasileiro para ser responsabilizada pela assistência a esse contingente populacional.

A alteração demográfica mais importante que influenciará o aumento da freqüência de utilização dos serviços de saúde é o rápido crescimento da proporção de pessoas com mais de 85 anos. Esse grupo apresenta geralmente uma grande carga de doenças crônicas e limitações funcionais.

De acordo com Hazzard et al. (1994), é significativo o efeito da idade avançada somado a certas condições causadoras de dependência muito freqüentes entre idosos, a saber demência, fraturas de quadril, acidentes vasculares cerebrais, doenças reumatológicas e deficiências visuais. Essas situações reduzem a capacidade do indivíduo de superar os desafios ambientais.

Observa-se que, com a falência do sistema previdenciário, a família vem progressivamente se tornando a única fonte de recursos disponível para o cuidado do idoso dependente. Saad (1991), em seu estudo sobre as tendências e conseqüências do envelhecimento populacional no Brasil, apresentado em um informe demográfico da Fundação Sistema Estadual de Análise de Dados de São Paulo (Fundação Seade), aponta que há uma carência de redes de suporte formais ao idoso. A autora afirma que, diante dos dados apresentados em seu estudo, fica claro que a tarefa de amparar os idosos está quase que exclusivamente sob a responsabi- lidade das famílias, já que a organização comunitária também se mostra bastante incipiente.

Outro estudo sobre o suporte domiciliar aos adultos com perda da independência, realizada de 1991 a 1995 no Município de São Paulo com famílias de baixa renda (Karsch, 1998), aponta que mais de $90 \%$ das famílias não receberam ajuda de serviços, organizações ou grupos voluntários e/ou agências particulares, mas cerca de $30 \%$ delas confirmaram que se pudessem receber esse tipo de auxílio ficariam satisfeitas.

Sobre a questão financeira, a mesma pesquisa mostra que mais de $90 \%$ dos cuidadores declararam que nem os pacientes nem eles próprios recebiam apoio financeiro de qualquer instituição, salvo, eventualmente, de familiares mais próximos. Mais de $40 \%$ dos cuidadores disseram precisar de apoios que não recebem, tais como orientações, apoio pessoal, consultas mais freqüentes, auxílio em transporte, etc.

As evidências empíricas mostram que as doenças causadoras de dependência geram gastos crescentes, cujo impacto na economia familiar ainda não é conhecido no Brasil. A necessidade de assistência permanente ao enfermo gera um custo elevado para os familiares, pois, atualmente, nenhum sistema de atenção à saúde prevê uma oferta suficiente dos serviços necessários a uma população portadora de dependências com crescimento exponencial.

A relevância econômica dos gastos exige uma análise acurada para fornecer aos formuladores das políticas de saúde informações com as quais possam traçar objetivos e programas de ação visando a uma solução qualitativa e economicamente viável para o problema. Hoje, pouco se conhece sobre o impacto econômico da dependência do idoso na família e no próprio sistema de saúde. Seria de fundamental importância para a formulação de políticas saber quais são os gastos de um paciente idoso com alto grau de dependência para o Sistema Único de Saúde, para os planos privados de assistência à saúde e para aqueles sem cobertura alguma.

No Brasil, é notória a inexistência de um programa de governo direcionado para a população idosa que desenvolve dependência, apesar da existência de uma Política Nacional de Saúde do Idoso (Brasil, 1999), cujo DecretoLei foi promulgado em 1999.

Assim, pesquisas, investigações e estudos que busquem atualizar os conhecimentos e procurem estabelecer informações sobre o problema são relevantes e podem se transformar em fontes de contribuição para a gestão dos formuladores da política sanitária, quer sejam 
públicos ou privados. Afinal, citando Mendes (2001:103): “a crescente demanda de assistência à saúde da faixa etária que irá mais crescer nesse século e a existência de alternativas de atenção colocam importantes questionamentos a quem formula, planeja, executa e, principalmente, a quem financia os serviços de saúde. Insistir exclusivamente no modelo hospitalar e asilar significa uma total falta de sintonia com o que está acontecendo no mundo, como um desprezo pela realidade do idoso no Brasil".

\section{Dependência e envelhecimento}

O termo "dependência" liga-se a um conceito fundamental na prática geriátrica: a "fragilidade". A fragilidade é definida por Hazzard et al. (1994) como uma vulnerabilidade que o indivíduo apresenta aos desafios do próprio ambiente. Essa condição é observada em pessoas com mais de 85 anos ou naqueles mais jovens que apresentam uma combinação de doenças ou limitações funcionais que reduzam sua capacidade de adaptar-se ao estresse causado por doenças agudas, hospitalização ou outras situações de risco.

Ao se definir o objeto "envelhecimento com dependência”, destaca-se a questão da prevalência de patologias crônicas nesse grupo etário. Llera \& Martín (1994) afirmam que essa cronicidade observada na maioria dos idosos necessita de uma abordagem abrangente para ser bem avaliada, já que as doenças crônicas podem ser incapacitantes ou não. Portanto, faz-se necessário classificar a incapacidade em graus de dependência: leve, parcial ou total. É exatamente o grau de dependência que determina os tipos de cuidados que serão necessários.

Para se avaliar o grau de dependência utiliza-se o método de avaliação funcional. Esse método, bastante conhecido e aplicado na prática geriátrica, tem se mostrado um indicador sensível e relevante para avaliar necessidades e determinar a utilização de recursos.

A “função" é definida como a capacidade de um indivíduo se adaptar aos problemas cotidianos, ou seja, aquelas atividades que lhe são requeridas por seu entorno imediato, incluindo a sua participação como indivíduo na sociedade, ainda que apresente alguma limitação física, mental ou social. É portanto um fenômeno complexo, influenciado por múltiplos fatores.

A função é avaliada com base na capacidade de execução das atividades da vida diária (AVD), que por sua vez se dividem em: (a) atividades básicas da vida diária - tarefas próprias do autocuidado, como alimentar-se, vestir-se, controlar os esfíncteres, banhar-se, locomoverse, etc.; (b) atividades instrumentais da vida diária - indicativas da capacidade para levar uma vida independente na comunidade, como realizar as tarefas domésticas, compras, administrar as próprias medicações, manusear dinheiro, etc.; e (c) atividades avançadas da vida diária - marcadoras de atos mais complexos, e em grande parte, ligados à automotivação, como trabalho, atividades de lazer, contatos sociais, exercícios físicos, etc.

É importante acrescentar, segundo Pitaud (1999), que a realização das atividades listadas acima, além da capacidade de participar da vida econômica e social e decidir o que se quer fazer com seus recursos sem a ajuda de terceiros, caracteriza uma pessoa independente.

A dependência se traduz por uma ajuda indispensável para a realização dos atos elementares da vida. Não é apenas a incapacidade que cria a dependência, mas sim o somatório da incapacidade com a necessidade. Por outro lado, a dependência não é um estado permanente. É um processo dinâmico cuja evolução pode se modificar e até ser prevenida ou reduzida se houver ambiente e assistência adequados.

\section{A questão econômica}

Chappell (1993), Kosberg (1992), Karsch (1998), Moragas (1994) e outros estudiosos do assunto apontam que, quando os idosos são acometidos por doenças incapacitantes, particularmente em relação àquelas oriundas da deterioração das funções neurológicas, causadoras de dependência em alto grau, observa-se que o cuidado tem sido prestado por um sistema de suporte informal, em especial nos países em desenvolvimento. Esse sistema inclui cuidadores que podem ser familiares, amigos, vizinhos e membros da comunidade cujas atividades são, em geral, prestadas voluntariamente, sem remuneração. Ramos et al. (1993), em estudo realizado no Município de São Paulo, demonstram que $2 \%$ dos idosos não contam com nenhuma ajuda familiar em caso de doença ou incapacidade; $40 \%$ contam com o cônjuge; $35 \%$ contam com a filha; $11 \%$, com o filho e $10 \%$, com toda a família. Nos domicílios unigeracionais cresce a perspectiva de ajuda do cônjuge $(60 \%)$ e, nos multigeracionais, da filha (56\%) e do filho (13\%). Yasaki et al. (1991) acrescentam que, embora os idosos pobres residentes em áreas metropolitanas com comprometimentos físicos e cognitivos estejam convivendo compulsoriamente com os filhos, o asilamento é 
visto como negativo tanto pelos próprios idosos quanto por seus familiares, de todos os níveis de renda.

Chappell (1993) refere que o cuidado informal é parte da abordagem mais atual da atenção social prestada na comunidade. Essa autora contribui com afirmações baseadas em pesquisas que comparam as políticas de atenção ao idoso em seu país, o Canadá, com as políticas de outros países desenvolvidos. Chappell (1993) então aponta que em todas as abordagens as contribuições e o papel do cuidado informal da família e dos amigos são uma pedra angular na nova retórica, apoiada pela pesquisa gerontológica sobre suporte e cuidado prestados aos idosos.

A família e os amigos são a primeira fonte de cuidados. O maior indicador para o asilamento e outras formas de institucionalização de longa duração entre idosos é a falta de suporte familiar e a inexistência de doença. Chappell (1993) acrescenta que, apesar das evidências a favor da promoção da saúde, da prevenção de doenças, da necessidade de um cuidado na comunidade (community care) e do desejo pela participação e integração do idoso na sociedade, os investimentos do governo federal do Canadá nessas áreas permanecem baixos. A maior parte dos investimentos ainda vai para o cuidado curativo.

Hoje já é reconhecida a importância de cuidado de longo prazo na comunidade para a população idosa. Chappell (1993) afirma que os países industrializados têm reconhecido essa importância e a integração dos cuidados comunitários ao sistema de saúde está acontecendo. Esses países também demonstram que ampliar a atenção à saúde para uma maior valorização e inserção de cuidados na comunidade é concomitante aos estudos e preocupações com custo-eficácia.

Segundo Moragas (1994) - teórico espanhol que se dedicou a estudar os diversos aspectos da dependência -, os responsáveis pelas áreas de saúde e social de diversos países afirmam que não existe numerário público capaz de fazer frente à totalidade das necessidades que os enfermos portadores de dependência precisarão para os próximos anos. Não obstante, existe certa reticência em planejar o futuro dessa assistência, uma vez que não desperta o interesse de políticos e os técnicos não têm soluções.

A importância econômica do gasto exige um planejamento múltiplo, já que é impensável que somente o sistema público possa fazer frente ao gasto com o cuidado aos doentes. A institucionalização de todos os afetados não é possível, seja por motivos de custo ou por mo- tivos de qualidade da assistência, portanto é importante questionar o próprio modelo de assistência, que deve priorizar a promoção da saúde visando a um envelhecimento bem-sucedido.

Walker (1990, apud Chappell, 1993), aponta que a impossibilidade de atender à demanda gerada pelo aumento da população idosa pode ser usada como justificativa para se repassar à família as responsabilidades e, então, as instâncias que poderiam respaldar o idoso desobrigam-se de investir nessa área.

As políticas públicas de vários países, incluindo o Brasil, reconhecem oficialmente a contribuição dos cuidadores informais, dos voluntários e do setor privado para complementar a assistência pública. Mas a questão do financiamento do cuidado comunitário permanece um desafio a ser enfrentado. Que parcela cabe às famílias no tratamento do paciente idoso dependente de cuidados? E qual é a parcela que cabe ao Estado?

A dependência precisa ser reconhecida como uma importante questão de saúde pública. Seu impacto sobre a família e a sociedade não pode ser subestimado. No Brasil, a Política Nacional de Saúde do Idoso (Brasil, 1999), reconhecendo a importância da parceria entre os profissionais de saúde e as pessoas que cuidam dos idosos, aponta que essa parceria deverá possibilitar a sistematização das tarefas a serem realizadas no próprio domicílio, privilegiandose aquelas relacionadas à promoção da saúde, à prevenção de incapacidades e à manutenção da capacidade funcional do idoso dependente e do seu cuidador, evitando-se assim, na medida do possível, hospitalizações, asilamento e outras formas de segregação e isolamento.

No entanto, essa política não foi regulamentada e, portanto, não foram abordadas questões relativas ao financiamento das ações previstas na política, tampouco explicitadas as estratégias a serem utilizadas para alcançar os objetivos supracitados.

\section{O cuidado familiar}

A família predomina como alternativa no sistema de suporte informal aos idosos. Kosberg (1992), em seu estudo comparativo sobre o cuidado familiar do idoso em diversos países, nos cinco continentes, aponta várias razões para que esta tenha se tornado a principal fonte de cuidados para os idosos.

Entretanto, é preciso destacar que, embora o cuidado familiar seja um aspecto importante, ele não se aplica a todos os idosos. Existem 
idosos que não têm família. Há outros cujas famílias são muito pobres ou seus familiares precisam trabalhar e não podem deixar o mercado de trabalho para cuidar deles.

O fato de morar só, para o idoso, tem sido associado a um decréscimo na qualidade de vida, agravamento da morbidade e, até mesmo, indicador de risco de mortalidade. De acordo com a Pesquisa Nacional de Saúde e Nutrição (PNSN) (INAN, 1990), mais de $10 \%$ da população idosa brasileira morava sozinha, com a maior proporção recaindo sobre as mulheres, em especial as de 80 anos e mais que residiam na zona rural (23\%). Portanto, diferentemente dos países norte-americanos e europeus, a maioria dos idosos brasileiros mora com a família.

Ainda baseada na PNSN, Anderson (1997) destaca, em seu estudo sobre saúde e condições de vida do idoso no Brasil, que grande parte da população, principalmente nos grandes centros urbanos, reside em domicílios com um número restrito de cômodos que, cada vez mais, diminuem de tamanho, comprometendo as condições de conforto e privacidade do idoso. Araújo \& Alves (2000) informam que, segundo o PNAD (IBGE, 1996), mais de 85\% dos idosos no Brasil vivem em domicílios onde existe a presença de parentes e somente uma pequena parte desses idosos $(11,6 \%)$ vive sozinha ou com pessoas sem nenhum laço de parentesco.

Estudos sobre transferências intergeracionais têm mostrado que, entre as famílias mais pobres, os idosos contribuem com o seu rendimento da aposentadoria para o orçamento domiciliar. Essa contribuição tem papel importante nas estratégias de sobrevivência do grupo doméstico. O importante estudo de Camarano (2002), baseado em dados do Instituto de Pesquisa Econômica Aplicada (IPEA), destaca essa questão.

No entanto, embora o idoso brasileiro nos estratos mais pobres da população contribua com sua renda para o sustento da família, nem sempre ele recebe o respaldo de que necessita, tanto por ser insuficiente o recurso quanto pelas dificuldades que a família encontra para dele cuidar. Caldas (2002) afirma que um cuidado que se apresenta de forma inadequada, ineficiente ou mesmo inexistente, é observado em situações nas quais os membros da família não estão disponíveis, estão despreparados ou estão sobrecarregados por essa responsabilidade. Nesse contexto, existe a possibilidade concreta de serem perpetrados abusos e maus-tratos. Portanto, é necessário lembrar que, embora a legislação e as políticas públicas afirmem e a própria sociedade acredite que os idosos de- vam ser cuidados pela família (por questões morais, econômicas ou éticas), não se pode garantir que a família prestará um cuidado humanizado.

Para acompanhar o fluxo de tais mudanças são imprescindíveis programas e serviços para idosos. Essas ações são urgentes e necessárias, pois muitos idosos isolados, dependentes e abandonados necessitam de alternativas à assistência familiar de que não dispõem.

\section{As necessidades da família}

Caldas (2000) e Harvis \& Rabins (1989) indicam que a família apresenta necessidades que vão desde os aspectos materiais até os emocionais, passando pela necessidade de informações. $\mathrm{O}$ aspecto material inclui recursos financeiros, questões de moradia, transporte e acesso a serviços de saúde. Por outro lado, essa família-cuidadora necessita de informação sobre como realizar os cuidados, incluindo a adaptação do ambiente ao idoso. Além disso, são importantes o suporte emocional, uma rede de cuidados que ligue a família aos serviços de apoio e meios que garantam qualidade de vida aos cuidadores principais.

Medeiros et al. (1998) abordam um aspecto de grande relevância para a questão do financiamento da assistência ao idoso dependente. Trata-se da transferência do papel da seguridade social para as famílias. Os benefícios previdenciários constituem o substitutivo da renda do trabalho humano, quando este não pode mais ser exercido diante do surgimento de um risco social ou profissional. Essa proteção social no Brasil está assegurada pela Previdência Social.

Medeiros et al. (1998:122) definem Previdência Social como “...um agente de equilíbrio social que tem o objetivo de assegurar recursos para a manutenção do indivíduo e seus familiares nos casos de riscos ou contingências sociais, determinados por morte, incapacidade, velhice, invalidez, desemprego ou reclusão". A autora prossegue afirmando que a garantia dessa proteção está em questão porque, em conseqüência da transição demográfica, houve um aumento da expectativa de vida e, conseqüentemente, uma ampliação do número de pessoas que recebem benefícios, sem que tenha aumentado, na mesma proporção, o número de contribuintes. Hoje, a proporção é preocupante: para cada 2,3 contribuintes existe uma pessoa recebendo benefício mensalmente.

Percebe-se então que a seguridade social, que objetiva garantir a saúde, a previdência e a 
assistência social (Constituição Federal - Brasil, 1988), não tem conseguido cumprir seu papel, transferindo-o para os cidadãos e fazendo recair sobre a família a cobertura de suas falhas. Por sua vez, a família desse paciente idoso dependente, que recebe os baixos benefícios previdenciários do paciente (aposentadoria, pensão ou benefício de auxílio-doença), não consegue arcar com os custos do cuidado.

Ainda que se considere a dificuldade de financiamento da assistência ao idoso dependente na comunidade, é possível estabelecer políticas públicas que envolvam mecanismos de apoio institucional e comunitário às famílias que cuidam e aos idosos que não possuem uma família que assuma os cuidados. Com base nos dados da pesquisa desenvolvida em sua tese de doutorado, Caldas (2000) concluiu que, quando contam com uma estrutura de apoio institucional, estratégico, material e emocional, os cuidadores têm a possibilidade de exercer o cuidado e permanecer inseridos socialmente sem imobilizar-se pela sobrecarga determinada pela difícil e estafante atenção ao doente dependente.

No entanto, é necessário aprofundar os estudos a respeito dessa realidade. Ao ressaltar a importância de estudos desse tipo, Karsch (1998) aponta que nos países desenvolvidos, onde existem estudos conclusivos sobre os custos da assistência hospitalar e do asilamento de pessoas que perderam sua capacidade funcional, os programas de community careque geralmente se baseiam no levantamento de recursos formais e informais existentes em bairros ou regiões das grandes cidades - têm sido as alternativas menos caras de assistência à saúde. Para tal finalidade, são incentivados estudos que revelem quem são as pessoas que cuidam de idosos mais frágeis, seja por doenças crônico-degenerativas, seja pela idade em si, que demandam atenção constante e medidas de follow-up após cada alta hospitalar.

O fato de um membro da família desencadear um processo de dependência altera a dinâmica familiar. À medida que a pessoa vai desenvolvendo a doença, há uma mudança de papéis nos membros da família. Mendes (1995) aponta que, em geral, a decisão de assumir os cuidados é consciente, e os estudos revelam que, embora a designação do cuidador seja informal e decorrente de uma dinâmica, o processo parece obedecer a certas regras refletidas em quatro fatores: parentesco, com freqüência maior para os cônjuges, antecedendo sempre a presença de algum filho; gênero, com predominância da mulher; proximidade física, considerando quem vive com a pessoa que requer cui- dados; e proximidade afetiva, destacando a relação conjugal e a relação entre pais e filhos.

Em geral, são as mulheres que assumem o cuidado, e esse papel é visto como natural, pois está inscrito socialmente no papel de mãe. Cuidar dos familiares idosos, portanto, é mais um dos papéis que a mulher assume na esfera doméstica. Nesse contexto, surge outra variável significativa: a faixa etária dos cuidadores que pertencem, freqüentemente, à mesma geração dos doentes. São "idosos jovens independentes" cuidando de "idosos dependentes".

De acordo com Caldas (1995), a sobrecarga física, emocional e sócio-econômica do cuidado de um familiar é imensa. E não se deve esperar que os cuidados sejam entendidos e executados corretamente sem que os responsáveis pelo paciente sejam orientados. Seria fundamental que profissionais de saúde treinassem o cuidador e supervisionassem a execução das atividades assistenciais necessárias ao cotidiano do idoso até que a família se sentisse segura para assumi-las. A família deve ser preparada também para lidar com os sentimentos de culpa, frustração, raiva, depressão e outros sentimentos que acompanham essa responsabilidade.

Medeiros et al. (1998) apontam os resultados de um estudo qualitativo sobre a trajetória de vidas de cuidadores de idosos altamente dependentes no Município de São Paulo, com uma população de baixa renda que pode ser um retrato bastante fidedigno de outras regiões metropolitanas do Brasil sobre a falta de apoio e orientação para cuidar.

Os cuidadores mencionaram que, quando da alta hospitalar, os familiares raramente receberam informações claras a respeito da doença, orientação ou apoio para os cuidados, nem indicação de um serviço para prosseguir o tratamento. A maioria desses cuidadores foi orientada, superficialmente, sobre medicação, alimentação e retornos.

O abandono, a falta de orientação e a falta de recursos estão presentes não só no momento da alta hospitalar, mas também no tratamento ambulatorial. A demora é grande para se conseguir marcar as consultas de acompanhamento médico, demora que é ainda maior quando é preciso consultar especialistas, exames complementares, fisioterapia e fonoterapia.

Muitas vezes, os familiares nem sequer recebem orientação sobre onde conseguir o tratamento necessário. Além do que, quando obtêm o recurso, falta-lhes transporte adequado para as limitações do paciente, uma vez que muitos têm dificuldades de locomoção. Esta- 
belece-se, então, um círculo vicioso: doença, falta de tratamento, agravamento da doença e, como conseqüência, aumento dos gastos com a doença.

Um estudo de referência nessa área de suporte aos cuidados domiciliares é descrito por Felgar (1998). Trata-se da pesquisa coordenada por Úrsula Karsch, denominada Estudo do Suporte Domiciliar aos Adultos com Perda da Independência e Perfil do Cuidador Principal. Realizada de 1991 a 1995 no Município de São Paulo, teve como objetivo conhecer as características dos cuidados comunitários oferecidos aos adultos com diferentes graus de dependência, de uma perspectiva longitudinal, em uma área metropolitana do Brasil.

A amostra inclui 160 pacientes que tiveram alta de diversos hospitais de São Paulo após internação por episódios agudos de AVC. Esses pacientes foram visitados três vezes ao longo de um ano. Os dados mostram que eram famílias de baixa renda. Cerca de $70 \%$ vivem com até cinco salários mínimos, dos quais quase $50 \%$, com até três salários mínimos.

Em relação ao impacto da doença sobre as condições materiais da família, observou-se que não há substituição de tarefas anteriores à tarefa de cuidar do idoso acometido por AVC. O desempenho cotidiano, seja no domicílio seja em trabalho extradomiciliar, vê-se acrescido da tarefa de cuidar. Aponte-se também a pequena extensão dos orçamentos, que passam a ser mais comprimidos pelas inesperadas despesas com hospitais, transporte, medicação, equipamentos, etc., como fator de sobrecarga para essas famílias.

Enfim, citando Karsch (1998), no atual quadro precário e insuficiente dos serviços do sistema brasileiro de saúde, constata-se que os velhos dependentes sobrevivem com poucos recursos pessoais e sociais. Longe do atendimento institucional, entretanto, encontram-se familiares, amigos e grupos religiosos, ou seja, formas de ajuda não visíveis de imediato, mas presentes nas dificuldades cotidianas, cumprindo o difícil papel de tecer a rede de cuidados, muitas vezes improvisados, que fornece algum suporte àqueles cuja gravidade de dependência exige que estejam sob a responsabilidade de outra pessoa.

\section{Considerações finais}

Cada vez mais o tema do envelhecimento com dependência vem sendo abordado nos países desenvolvidos. No Brasil, o envelhecimento da população é um fenômeno relativamente re- cente, no entanto já existem muitos estudos que apontam, de forma recorrente, que esse é um processo irreversível, diante do comportamento da fecundidade e da mortalidade registrado nas últimas décadas e do esperado para as próximas.

Observa-se que as condições e a qualidade de vida da população idosa no Brasil já eram precárias quando evidenciadas pelos dados da PNSN em 1990. Ao consultar dados da PNAD de 1996, nota-se que alguns indicadores apresentaram melhora, como o nível de escolaridade e o índice de Gini.

A questão é que, paralelamente ao aumento do número de idosos na população, convivemos com os efeitos sociais da crise econômica que o Brasil enfrenta. A recessão econômica traz profundos impactos sociais sobre a vida dos idosos, particularmente para aqueles que apresentam alguma dependência. As políticas de ajuste têm contribuído para agravar esses impactos, acentuando as desigualdades.

Mesmo com a conquista da renda pela assistência social para o idoso pobre garantida pela Constituição Federal (Brasil, 1988), o problema do idoso dependente não foi resolvido, pois, com o alto índice de desemprego, o rendimento do idoso passa a sustentar a família e com isso, a renda familiar mantém-se abaixo da linha de pobreza.

Portanto, ao analisarmos tanto os dados da PNSN de 1990 quanto os da PNAD de 1996, concluímos que o baixo nível de renda, a baixa escolaridade e a carência de serviços públicos e de saneamento básico são indicadores de precariedade das condições e da qualidade de vida para a população idosa no Brasil, em especial na zona rural. É preciso inserir a questão do envelhecimento com dependência nesse quadro que, por si, já é preocupante.

A dependência de um familiar idoso gera impacto na dinâmica, na economia familiar e na saúde dos membros da família que se ocupam dos cuidados. Por outro lado, é necessário pensar nos idosos que não têm uma família para assumir os cuidados necessários em situações de dependência.

Embora a Constituição Federal (Brasil, 1988), a Política Nacional do Idoso (Brasil, 1994) e a Política Nacional de Saúde do Idoso (Brasil, 1999) apontem a família como responsável pelo o atendimento às necessidades do idoso, até agora o delineamento de um sistema de apoio às famílias e a definição das responsabilidades das instâncias de cuidados formais e informais, na prática, não aconteceram. O sistema de saúde, público ou privado, não está preparado para atender nem a demanda de idosos que cres- 
ce a cada dia, nem a de seus familiares. Por sua vez o sistema previdenciário, público ou privado, não prevê formas de financiamento para o estabelecimento de redes de apoio às necessidades de assistência aos idosos dependentes, com ou sem família.

A dependência deve ser destacada como um processo dinâmico. Sua evolução pode modificar-se ou até ser prevenida se houver ambiente e assistência adequados. Portanto, a solução do problema representado pelo envelhecimento com dependência inclui o delineamento de uma política que envolva todos os setores da sociedade, e não apenas o governo, e o estabelecimento de programas que atendam aos idosos independentes a fim de prevenir a

\section{Referências}

ANDERSON, M. I. P., 1997. Saúde e Condições de Vida do Idoso no Brasil. Dissertação de Mestrado, Rio de Janeiro: Instituto de Medicina Social, Universidade do Estado do Rio de Janeiro.

ARAUJO, T. C. N. \& ALVES, M. I. C., 2000. Perfil da população idosa no Brasil. Textos sobre o Envelhecimento, 3:7-19.

BRASIL, 1988. Constituição da República Federativa do Brasil. Brasília: Senado Federal.

BRASIL, 1994. Lei no 8.842, de 4 de janeiro de 1994. Dispõe sobre a Política Nacional do Idoso, cria o Conselho Nacional do Idoso e dá outras providências. Brasília: Diário Oficial da República Federativa do Brasil, vol. 132, n. 3, pp. 77-79, Seção 1 , pt. 1.

BRASIL, 1999. Política Nacional de Saúde do Idoso, aprovada pela Portaria no 1.395, de 9 de dezembro de 1999. Brasília: Diário Oficial da República Federativa do Brasil, no 237-E, pp. 20-24, 13 dez. Seção 1.

CALDAS, C. P., 1995. A abordagem do enfermeiro na assistência ao cliente portador de demência. Revista Enfermagem da UERJ, 3:2.

CALDAS, C. P., 2000. O Sentido do ser Cuidando de uma Pessoa Idosa que Vivencia um Processo Demencial. Tese de Doutorado, Rio de Janeiro: Escola de Enfermagem Anna Nery, Universidade Federal do Rio de Janeiro. dependência. E isso deve ser parte dos programas de promoção da saúde.

O Programa Saúde da Família pode ser uma estratégia eficiente para fazer face a esse desafio, mas seria necessário que a questão do cuidado ao idoso dependente fosse incorporada pelo programa de forma específica, incluindo previsão de financiamento das ações e estabelecimento de uma rede de suporte institucional, o que ainda não foi feito. O cuidador informal poderia e deveria ser visto como um agente de saúde e receber orientações direcionadas para prestar um cuidado adequado ao idoso, incluindo medidas preventivas para evitar a dependência precoce e específicas sobre os cuidados com o idoso dependente que envelhece na comunidade.

CALDAS, C. P., 2002. O idoso em processo demencial: o impacto na família. In: Antropologia, Saúde e Envelhecimento (M. C. S. Minayo \& C. Coimbra Jr., org.), pp. 51-71, Rio de Janeiro: Editora Fiocruz.

CAMARANO, A. A., 1999. Como vive o idoso brasileiro? In: Muito Além dos 60: Os Novos Idosos Brasileiros (A. A. Camarano, org.), pp. 19-71, Rio de Janeiro: Instituto de Pesquisa Econômica Aplicada.

CHAPPELL, N. L., 1993. The future of health care in Canada. Journal of Social Policy, 22:487-505.

FELGAR, J. A. S., 1998. Uma expressão da linguagem numérica In: Envelhecimento com Dependência: Revelando Cuidadores (U. M. S. Karsch, org.), pp. 47-85, São Paulo: EDUC.

HARVIS, K. A. \& RABINS, P. V., 1989. Dementia. Helping family caregivers cope. Journal of Psychosocial Nursing, 27:5.

HAZZARD, W. R.; BRERMAN, E. L.; BLASS, J. P.; ETTINGER, W. H. \& HALTER, J. B., 1994. Principles of Geriatric Medicine and Gerontology. 3rd Ed. New York: McGraw Hill.

IBGE (Fundação Instituto Brasileiro de Geografia e Estatística), 1996. Pesquisa Nacional por Amostra de Domicílios (PNAD): Síntese de Indicadores. Rio de Janeiro: IBGE.

INAN (Instituto Nacional de Alimentação e Nutrição), 1990. Pesquisa Nacional sobre Saúde e Nutrição (PNSN). Brasília: Secretaria de Projetos Especiais. 
KARSCH, U. M. S., 1998. Envelhecimento com Dependência: Revelando Cuidadores. São Paulo: EDUC.

KOSBERG, J. I., 1992. Family Care of the Elderly. Thousand Oaks: Sage Publications.

LLERA, F. G. \& MARTIN, J. P. M., 1994. Síndromes y Cuidados en el Paciente Geriátrico. Barcelona: Masson.

MEDEIROS, S. A. R. R., VELAZQUEZ, M. D.; DAL RIO, M. C. \& MARQUES, D. D., 1998. As trajetórias de vida dos cuidadores principais. In: Envelhecimento com Dependência: Revelando Cuidadores (U. M. S. Karsch, org.), pp. 87-145, São Paulo: EDUC.

MENDES, P. B. M. T., 1995. Cuidadores: Heróis Anônimos do Cotidiano. Dissertação de Mestrado, São Paulo: Programa em Serviço Social, Pontifícia Universidade Católica de São Paulo.

MENDES, W., 2001. Home Care: Uma Modalidade de Assistência à Saúde. Rio de Janeiro: Universidade Aberta da Terceira Idade, Universidade do Estado do Rio de Janeiro.

MORAGAS, R. M., 1994. El Centro de Día para Enfermos de Alzheimer. Barcelona: Ucb Pharma.

PITAUD, P., 1999. La dependencia y su prise en charge (responsabilidad) en Francia. In: El Reto de la Dependencia al Envejecer (R. M. Moragas, org.), pp. 17-30, Barcelona: Herder.
RAMOS, L. R.; ROSA, E. C.; OLIVEIRA, Z. M.; MEDINA, M. C. G. \& SANTOS, F. R. G., 1993. Perfil do idoso em área metropolitana na região sudeste do Brasil: Resultados de inquérito domiciliar. Revista de Saúde Pública, 27:87-94.

SAAD, S. M., 1991. Tendências e conseqüências do envelhecimento populacional no Brasil. In: Série Informe Demográfico. A População Idosa e o Apoio Familiar (Fundação Sistema Estadual de Análise de Dados - SEADE, org.), pp. 3-10, São Paulo: Fundação SEADE.

WALKER, A., 1990. The Economic Burden of Aging and the Prospect of Intergenerational Conflict. Sheffield: Departament of Sociological Studies, University of Sheffield. (mimeo.)

YAZAKI, L. M.; MELO, A. V. \& RAMOS, L. R., 1991. Perspectivas atuais do papel da família frente ao envelhecimento populacional: Um estudo de caso. In: Série Informe Demográfico. A População Idosa e o Apoio Familiar (Fundação Sistema Estadual de Análise de Dados - SEADE, org.), pp. 11-26, São Paulo: Fundação SEADE.

Recebido em 25 de março de 2002

Versão final reapresentada em 8 de julho de 2002

Aprovado em 14 de agosto de 2002 